\title{
Xanthogranulomatous cholecystitis undergone laparotomy may go through troublesome clinical course: Reflection of severe inflammation
}

\author{
Hee Ju KIM, Kwangyeol PAIK*
}

Department of HBP Surgery, Yeoiudo St. Mary's Hospital, College of Medicine, The Catholic University of Korea, Seoul, Korea

Introduction: Xanthogranulomatous cholecystitis (XGC) show aggressive behavior which is indistinguishable from those of gallbladder cancer. Sometimes it was suspected simple cholecystitis and diagnosed only after surgery. The aim of current study is to present the perioperative clinical outcome according to the surgical methods for XGC which may reflect inflammatory degree.

Methods: Of 21 patients who underwent surgery at out hospital between January 2011 and October 2020, retrospective analysis was conducted to compare clinical outcomes according to the type of surgery which correlates with degree of inflammation.

Results: 21 patients were divided into two groups with regards to laparoscopic completion. Among them, thirteen underwent laparoscopic surgery (61.9\%) and eight underwent radical surgery under laparotomy (38.1\%). Laparotomy group was more suspected XGC or gallbladder cancer and laparoscopic group diagnosed more cholecystitis preoperatively. Laparotomy group needed more aggressive resection and showed higher intraoperative bleeding, conversion rate, longer operation time, longer stay in hospital and higher readmission rate perioperatively. Overall complication rate was not different on both groups.

Conclusions: Whether laparoscopic surgery is possible in the treatment of XGC reflects the acceptable clinical course. The patients undergone laparotomy need more comprehensive management even after surgery. Laparotomy with extended surgery may offer poorer intraoperative, perioperative, and short-term surgical outcomes rather than laparoscopic surgery for XGC which may reflect aggressiveness of it. 